\title{
Regulation of Autophagy of Prostate Cancer Cells by $\beta$-Catenin Signaling
}

\author{
Rongkai Lina Jiayu Feng ${ }^{\mathrm{b}}$ Shaoliang Donga Runyang Pan ${ }^{\mathrm{a}}$ Huiqiang Zhuang ${ }^{\mathrm{a}}$ \\ Zhenyu Ding ${ }^{c}$
}

aDepartment of Urology Surgery, No. 180 Hospital of PLA, Quanzhou, 'Department of Urology, Xinqiao Hospital, the Third Military Medical University, Chongqing, 'Department of Oncology, General Hospital of Shenyang Military Region, Shenyang, China

\section{Key Words}

$\beta$-catenin $\cdot$ Wnt $•$ Prostate cancer $•$ Autophagy $•$ Nitric oxide

\begin{abstract}
:
Background/Aims: Autophagy is a cellular degradation process for the recycling of damaged or superfluous intracellular compartments to provide an alternative energy source during periods of metabolic stress for maintaining cell homeostasis and viability. Although autophagy in different contexts have been shown to use similar signaling pathways, the exact molecular regulation of autophagy has been found to be cell-type dependent. Methods: We used rapamycin to trigger autophagy and used nitric oxide (NO) to inhibit autophagy in prostate cancer cells. IWP-2 was used to inhibit $\beta$-catenin signaling. Autophagy-associated proteins were examined by Western blot. Results: We found that nitric oxide (NO), a potent cellular messenger, impaired rapamycin-induced autophagy in prostate cancer cells. Further analyses showed that NO induced nuclear accumulation of $\beta$-catenin, a key factor of Wnt signaling pathway, to inhibit autophagy in prostate cancer cells. Conclusions: We demonstrate involvement of $\beta$-catenin signaling in the regulation of autophagy of prostate cancer cells. Our results shed light on a previously unappreciated $\beta$-catenin signaling pathway for regulating autophagy in prostate cancer.
\end{abstract}

Rongkai Lin and Zhenyu Ding

KARGER 125
Department of Urology Surgery, No. 180 Hospital of PLA, 180 Garden Road, Quanzhou 362000 (China) and Department of Oncology, General Hospital of Shenyang Military Region, 83 Wenhua Road, Shenyang 110840 (China)

E-Mail rongkailin180@gmail.com, rongkai_lin@163.com,E-Mail zhenyuding003@sina.com 


\section{Introduction}

Prostate cancer is one of the most common cancers in aged men [1-4]. Although our understanding and treatment of prostate cancer have significantly improved in the past years, the molecular regulation of the growth of prostate cancer cells is not yet clarified [1-6].

Autophagy is a catabolic pathway to degrade and recycle cellular compartments to promote cell survival at nutrient deprivation on physiological cellular metabolism [7]. However, autophagy also plays a critical role in carcinogenesis in that it regulates tumor suppression and therapeutic resistance as a survival mechanism in the harsh environment $[8,9]$. Since the molecular regulation of autophagy is cell-type dependent, it is necessary to examine autophagy in different tumor types.

Microtubule-associated protein 1A/1B-light chain 3 (LC3) is a soluble cellular protein. During autophagy, autophagosomes engulf cytoplasmic components [7-9]. Concomitantly, a cytosolic form of LC3 (LC3-I) is conjugated to phosphatidylethanolamine to form LC3phosphatidylethanolamine conjugate (LC3-II), which is then recruited to autophagosomes to form autolysosomes [7-9]. Afterwards, LC3-II in autolysosomal lumen is degraded with autolysosomes [7-9]. Thus, the autophagosomal marker LC3-II well reflects the autophagic activity [7-9].

The different signal transduction pathways and their crosstalk are critical for the cell fate decision, including autophagy, apoptosis, necrosis or necroptosis. However, our knowledge on these contexts is rather limited. Recent studies have shown that Wnt/ $\beta$ catenin signaling plays a role in autophagy in some models [10-15], but the involvement of this pathway in prostate cancer has not been reported. The Wnt signaling pathways are a group of signaling pathways that have been catalogued as the canonical Wnt pathway, the noncanonical planar cell polarity pathway and the noncanonical Wnt/calcium pathway. All three Wnt signaling pathways are activated by binding of a Wnt-protein ligand to a Frizzled family receptor, which transduces the biological signal into the cell. $\beta$-catenin is a key factor of the canonical Wnt pathway, and its nuclear translocation and retention lead to regulation of gene transcription [16].

Here we examined the role of $\beta$-catenin signaling in prostate cancer cells that were treated either with rapamycin as an autophagy inducer, or nitric oxide (NO) as an autophagy inhibitor. We found that NO significantly inhibited rapamycin-induced autophagy in prostate cancer cells via induction of $\beta$-catenin nuclear accumulation.

\section{Materials and Methods}

\section{Cell lines and reagents}

Human prostate cancer cell line PC3 and DU145 were purchased from ATCC (American Type Culture Collection, Manassas, VA, USA), and have been described before [17, 18]. PC3 cells were maintained in Dulbecco's modified Eagle's medium (DMEM, Invitrogen, Carlsbad, CA, USA) supplemented with 10\% fetal bovine serum (Invitrogen). DETA NONOate (a NO releasing compound, Sigma-Aldrich, St Louis, MO, USA), rapamycin (Sigma-Aldrich) and IWP-2 [19] (Santa Cruz, St Jose, CA, USA) are used in 500 $\mathrm{mol} / \mathrm{l}, 200 \mathrm{nmol} / \mathrm{l}$, $1 \mu \mathrm{mol} / \mathrm{l}$, respectively.

\section{Western blot}

Nuclear and cytoplasmic proteins were isolated with Nuclear and Cytoplasmic Extraction Kit (Thermo Scientific, Waltham, MA, USA). The protein from total cells was extracted from the cultured cells, which were homogenized in RIPA lysis buffer (1\% NP40, 0.1\% Sodium dodecyl sulfate (SDS), $100 \mu \mathrm{g} / \mathrm{ml}$ phenylmethylsulfonyl fluoride, $0.5 \%$ sodium deoxycholate, in PBS) on ice. The supernatants were collected after centrifugation at $12000 \times \mathrm{g}$ at $4^{\circ} \mathrm{C}$ for $20 \mathrm{~min}$. Protein concentration was determined using a BCA protein assay kit (Bio-rad, China), and whole lysates were mixed with $4 \times$ SDS loading buffer $(125 \mathrm{mmol} / \mathrm{l}$ Tris- $\mathrm{HCl}$, $4 \%$ SDS, 20\% glycerol, $100 \mathrm{mmol} / \mathrm{l}$ Dithiothreitol (DTT), and $0.2 \%$ bromophenol blue) at a ratio of 1:3. Samples were heated at $100{ }^{\circ} \mathrm{C}$ for $5 \mathrm{~min}$ and were separated on SDS-polyacrylamide gels. The separated 
Fig. 1. NO inhibited rapamycin-induced autophagy in prostate cancer cells. We used rapamycin as an autophagy inducer, and use NO as an autophagy inhibitor. (AE) Western blotting for Beclin-1, ATG7, p62 and autophagosome marker LC3 in rapamycin- and NO- treated prostate cancer cells, by representative Western blotting images (A), and by quantification (B-E). $* \mathrm{p}<0.05$.

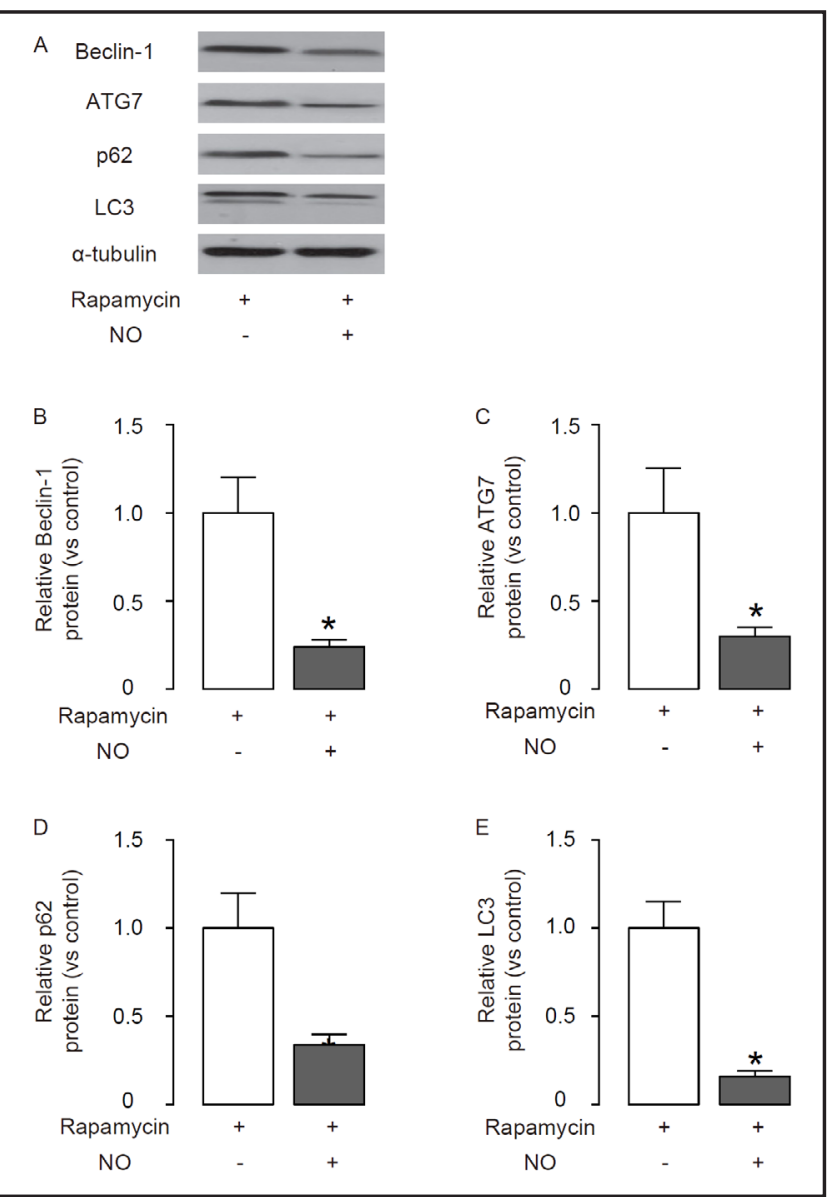

proteins were then transferred to a PVDF membrane. The membrane blots were first probed with a primary antibody. After incubation with horseradish peroxidase-conjugated second antibody, autoradiograms were prepared using the enhanced chemiluminescent system to visualize the protein antigen. The signals were recorded using X-ray film. Primary antibodies were anti-human $\beta$-catenin, Beclin-1, autophagy-related protein 7 (ATG7), p62, LC3, LaminB1 and $\alpha$-tubulin (all purchased from Cell Signaling, St Louis, MO, USA). $\alpha$-tubulin was used as a loading control for cytosol protein or total protein, and LaminB1 was used as a loading control for nuclear protein.

\section{Statistical analysis}

All statistical analyses were carried out using the SPSS 16.0 statistical software package. All values are depicted as mean \pm standard deviation from 5 individuals and are considered significant if $\mathrm{p}<0.05$. All data were statistically analyzed using one-way ANOVA with a Bonferoni correction.

\section{Results}

NO inhibited rapamycin-induced autophagy in prostate cancer cells

We first examined the potential of using rapamycin as an autophagy inducer, and the potential of using DETA NONOate (a NO releasing compound) as an autophagy inhibitor. We found that NO significantly decreased the levels of Beclin-1, ATG7, p62 and autophagosome marker LC3 in rapamycin-treated prostate cancer cells, by representative Western blotting images (Fig. 1A), and by quantification (Fig. 1B-E). These data confirmed the anti-autophagy role of NO in prostate cancer cells. 
Fig. 2. NO induced $\beta$-catenin nuclear accumulation in rapamycin-treated prostate cancer cells. Protein was isolated from cytosol and nucleus of prostate cancer cells. (A-B) Western blotting for $\beta$-catenin, LaminB1 (nuclear protein marker) and $\alpha$-tubulin (cytoplasmic protein marker) in rapamycin-, with/without NO-, with/ without IWP2- treated prostate cancer cells, by representative Western blotting images (A), and by quantification (B). ${ }^{*} \mathrm{p}<0.05$.

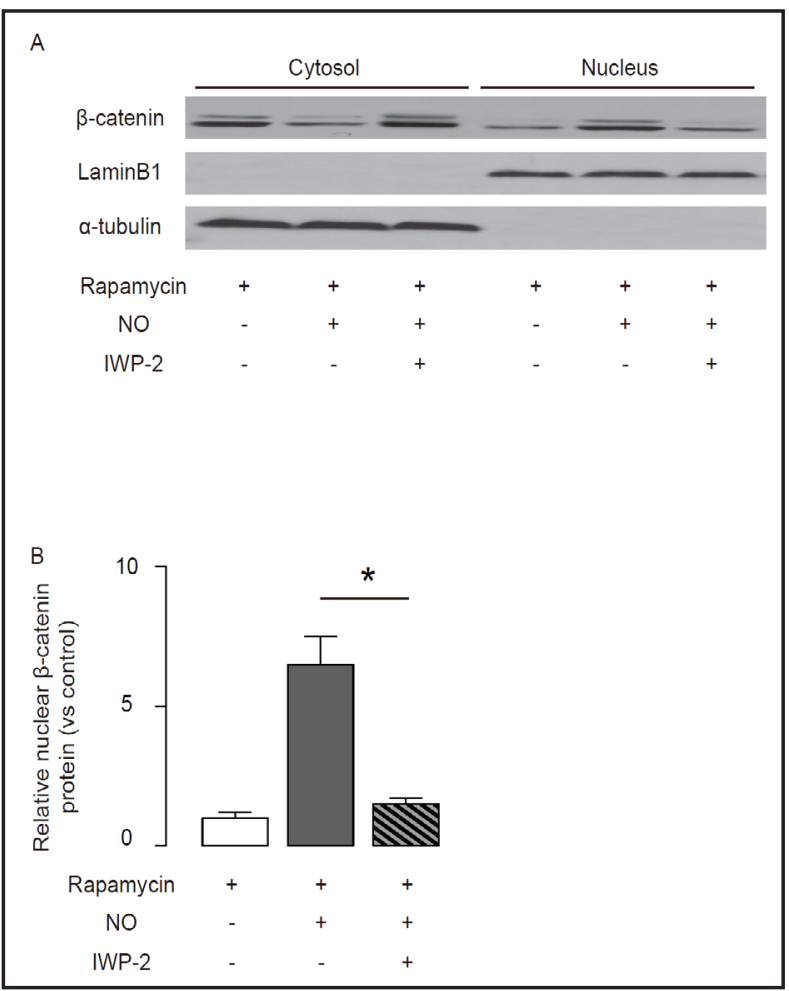

Fig. 3. NO inhibited autophagy of prostate cancer cells through $\beta$-catenin signaling. (A-E) Western blotting for Beclin-1, ATG7, p62 and autophagosome marker LC3 in rapamycin-, with/without NO-, with/without IWP2- treated prostate cancer cells, by representative Western blotting images (A), and by quantification (B-E). $* \mathrm{p}<0.05$.

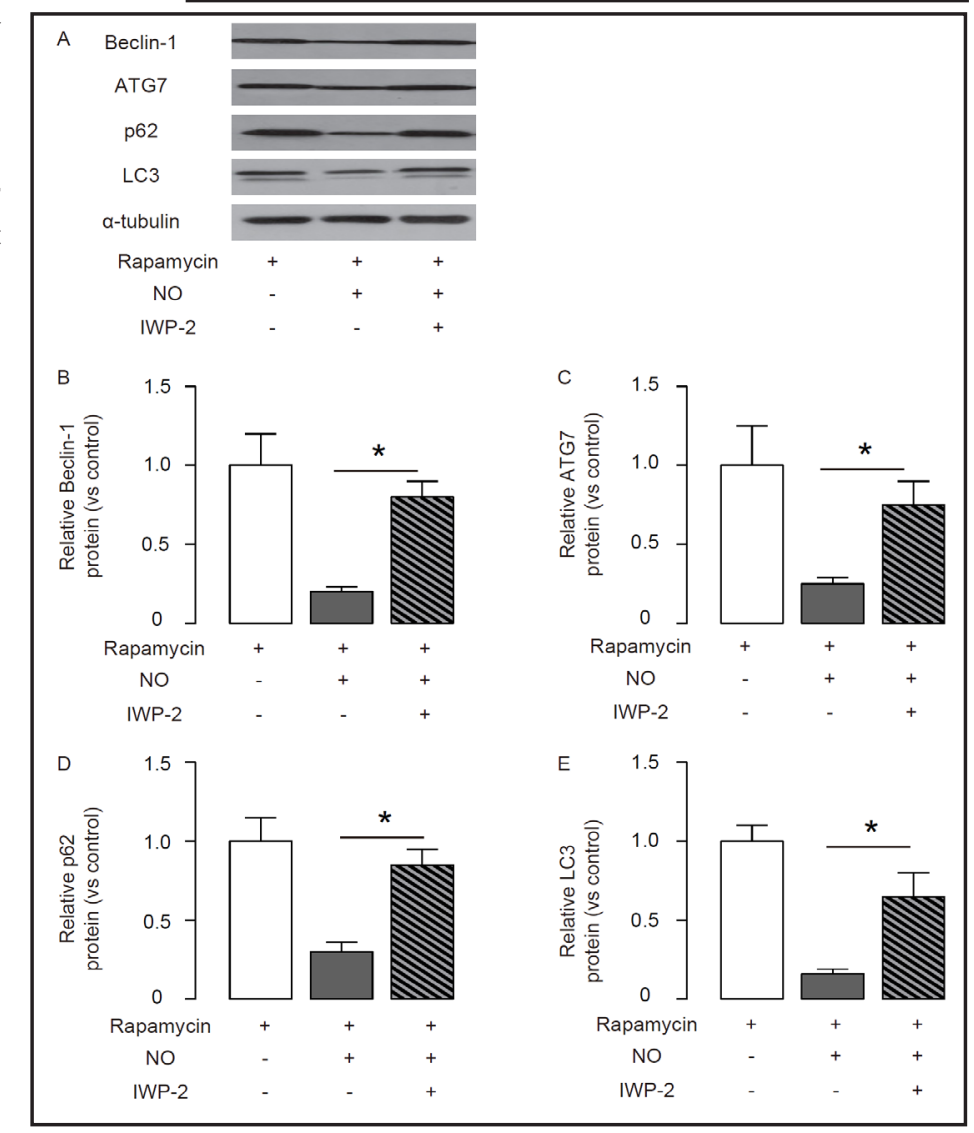

NO induced nuclear $\beta$-catenin accumulation in rapamycin-treated prostate cancer cells

We then examined the changes in $\beta$-catenin in prostate cancer cells induced by rapamycin and NO. $\beta$-catenin is regulated by phosphorylation. When Wnt signaling is not KARGER 
Fig. 4. Schematic of the model. NO inhibits autophagy of prostate cancer cells through enhancing $\beta$-catenin signaling.

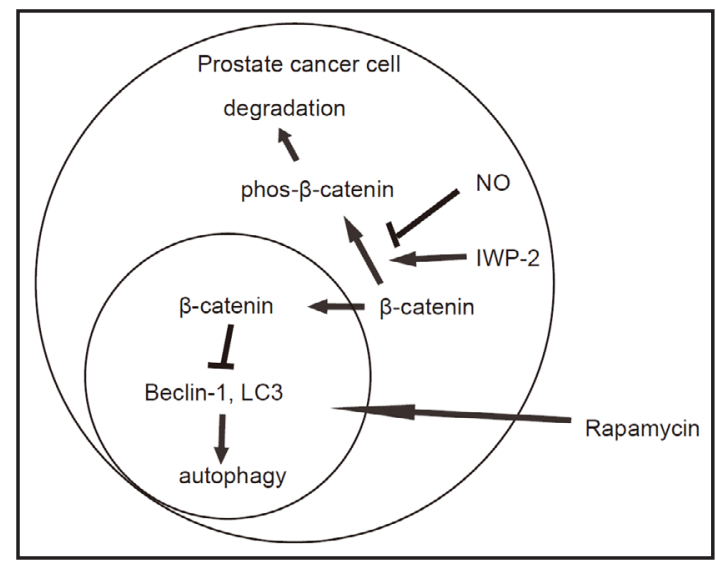

activated, $\beta$-catenin can be phosphorylated in the cytosol to undergo degradation. When Wnt signaling is activated, the phosphorylation of $\beta$-catenin is thus inhibited. Then the nuclear translocation of $\beta$-catenin will occur, resulting in nuclear accumulation of $\beta$-catenin to exert biological functions $[20,21]$. Since nuclear $\beta$-catenin is the functional status for $\beta$-catenin, we thus analyzed nuclear proteins and cytosol protein separately. We found that NO induced a significant increase in cytosol $\beta$-catenin in rapamycin-treated prostate cancer cells, by representative images (Fig. 2A), and by quantification (Fig. 2B). These data suggest that NO may regulate $\beta$-catenin signaling to modulate rapamycin-induced autophagy in prostate cancer cells.

NO inhibited autophagy of prostate cancer cells through $\beta$-catenin nuclear accumulation

In order to find out whether NO may inhibit the autophagy in rapamycin-treated prostate cancer cells through induction of $\beta$-catenin nuclear accumulation, we inhibited nuclear translocation of $\beta$-catenin in prostate cancer cells by a specific inhibitor IWP-2 [19]. The inhibition efficiency was confirmed by Western blotting, showing by representative images (Fig. 2A), and by quantification (Fig. 2B). We found that inhibition of $\beta$-catenin nuclear accumulation in prostate cancer cells completely abolished the inhibitory effect of NO on rapamycin-induced autophagy in prostate cancer cells (Fig. 3A-E). These data suggest that NO may inhibit autophagy of prostate cancer cells through enhancing $\beta$-catenin signaling. This model was thus summarized in a schematic (Fig. 4).

\section{Discussion}

Many oncogenes and tumor suppressors change expression levels in various tumors, and the presence of these oncogenic abnormalities is often associated with signaling pathways that regulate cell growth, cell metabolism and cell death. Thus, understanding the molecular regulation of different metabolic pathways in tumors may contribute to the identification of novel therapeutic targets and the development of more effective cancer therapies. Although altered metabolism may be beneficial to the cancer cells, it can create an increased demand for nutrients to support cell growth and proliferation, which creates metabolic stress and subsequently induces autophagy, a catabolic process leading to degradation of cellular components through the lysosomal system. Cancer cells use autophagy as a survival strategy to provide essential biomolecules that are required for cell viability under metabolic stress. Thus, autophagy appears to play a key role in the suppression of tumorigenesis. Understanding the context-dependent role of autophagy in cancer development should present new opportunities for the design of cancer therapeutics.

The involvement of different signal transduction pathways in autophagy is critical for the cell fate decision at the crossroads for autophagy, apoptosis, necrosis or necroptosis, since these pathways regulate all these biological events. Recent studies have shown that 
$\beta$-catenin signaling may play a role in autophagy in some models [10-15], whereas its involvement in autophagy in prostate cancer cells has not been addressed.

Here we examined the role of $\beta$-catenin signaling in autophagy of prostate cancer cells. Rapamycin is an inhibitor of the Ser/Thr protein kinase named "mammalian target of rapamycin" (mTOR) that regulates cell growth and metabolism in response to environmental cues. Rapamycin is also an inducer of autophagy, as inhibition of mTOR mimics cellular starvation by blocking signals required for cell growth and proliferation [22]. NO is an autophagy inhibitor, and has been shown to impair autophagy by inhibiting the activity of S-nitrosylation substrates, JNK1 and IKK $\beta$, by reducing Bcl-2 phosphorylation [23]. However, the exact molecular mechanism of the effect of NO on autophagy of prostate cancer cells remains not elucidated. We found that NO significantly inhibited rapamycininduced autophagy in prostate cancer cells, by examining the levels of all autophagy related proteins, including Beclin-1, ATG7, p62 and autophagosome marker LC3. Moreover, NO appeared to inhibit the autophagy in rapamycin-treated prostate cancer cells through $\beta$-catenin nuclear accumulation, since inhibition of $\beta$-catenin nuclear accumulation in prostate cancer cells completely abolished the inhibitory effect of NO on rapamycin-induced autophagy of prostate cancer cells. These data thus highlight a role of $\beta$-catenin signaling in the anti-autophagy effect of NO on prostate cancer cells. Moreover, we also checked another prostate cancer cell lines, DU145, and essentially got same results. Both DU145 and PC3 are the "classical" cell lines of human prostatic cancer. DU145 cells have moderate metastatic potential compared to PC3 cells which have high metastatic potential. Therefore, a cell-line dependent possibility was excluded. Our study thus offers new insights on the control of autophagy in prostate cancer cells.

\section{Disclosure Statement}

The authors have declared that no conflict of interest exists.

\section{Reference}

1 Saylor PJ: Prostate cancer: The androgen receptor remains front and centre. Nat Rev Clin Oncol 2013;10:126-128.

2 Alva A, Hussain M: The changing natural history of metastatic prostate cancer. Cancer J 2013;19:19-24.

-3 Beltran H, Rubin MA: New strategies in prostate cancer: Translating genomics into the clinic. Clin Cancer Res 2013;19:517-523.

4 Xin L: Cells of origin for cancer: An updated view from prostate cancer. Oncogene 2013;32:3655-3663.

5 Huang S, Liao Q, Li L, Xin D: Pttg1 inhibits smad3 in prostate cancer cells to promote their proliferation. Tumour Biol 2014;35:6265-6270.

6 Xia Q, Li C, Bian P, Wang J, Dong S: Targeting smad3 for inhibiting prostate cancer metastasis. Tumour Biol 2014;35:8537-8541.

7 Green DR, Levine B: To be or not to be? How selective autophagy and cell death govern cell fate. Cell 2014;157:65-75.

-8 Guo JY, Xia B, White E: Autophagy-mediated tumor promotion. Cell 2013;155:1216-1219.

1 White E: Deconvoluting the context-dependent role for autophagy in cancer. Nat Rev Cancer 2012;12:401410.

10 Jia Z, Wang J, Wang W, Tian Y, XiangWei W, Chen P, Ma K, Zhou C: Autophagy eliminates cytoplasmic betacatenin and nicd to promote the cardiac differentiation of p19cl6 cells. Cell Signal 2014;26:2299-2305.

11 Fu Y, Chang H, Peng X, Bai Q, Yi L, Zhou Y, Zhu J, Mi M: Resveratrol inhibits breast cancer stem-like cells and induces autophagy via suppressing wnt/beta-catenin signaling pathway. PLoS One 2014;9:e102535.

12 Liu D, Cui W, Liu B, Hu H, Liu J, Xie R, Yang X, Gu G, Zhang J, Zheng H: Atorvastatin protects vascular smooth muscle cells from tgf-beta1-stimulated calcification by inducing autophagy via suppression of the betacatenin pathway. Cell Physiol Biochem 2014;33:129-141. 
13 Petherick KJ, Williams AC, Lane JD, Ordonez-Moran P, Huelsken J, Collard TJ, Smartt HJ, Batson J, Malik K, Paraskeva C, Greenhough A: Autolysosomal beta-catenin degradation regulates wnt-autophagy-p62 crosstalk. EMBO J 2013;32:1903-1916.

14 Chang HW, Lee YS, Nam HY, Han MW, Kim HJ, Moon SY, Jeon H, Park JJ, Carey TE, Chang SE, Kim SW, Kim SY: Knockdown of beta-catenin controls both apoptotic and autophagic cell death through lkb1/ampk signaling in head and neck squamous cell carcinoma cell lines. Cell Signal 2013;25:839-847.

15 Nguyen TM, Subramanian IV, Xiao X, Ghosh G, Nguyen P, Kelekar A, Ramakrishnan S: Endostatin induces autophagy in endothelial cells by modulating beclin 1 and beta-catenin levels. J Cell Mol Med 2009;13:3687-3698.

-16 Clevers H, Nusse R: Wnt/beta-catenin signaling and disease. Cell 2012;149:1192-1205.

17 Huang SQ, Liao QJ, Wang XW, Xin DQ, Chen SX, Wu QJ, Ye G: Rnai-mediated knockdown of pituitary tumortransforming gene-1 (pttg1) suppresses the proliferation and invasive potential of pc3 human prostate cancer cells. Braz J Med Biol Res 2012;45:995-1001.

18 Durocher Y, Chapdelaine A, Chevalier S: Tyrosine protein kinase activity of human hyperplastic prostate and carcinoma cell lines pc3 and du145. Cancer Res 1989;49:4818-4823.

19 Chen B, Dodge ME, Tang W, Lu J, Ma Z, Fan CW, Wei S, Hao W, Kilgore J, Williams NS, Roth MG, Amatruda JF, Chen C, Lum L: Small molecule-mediated disruption of wnt-dependent signaling in tissue regeneration and cancer. Nat Chem Biol 2009;5:100-107.

20 Holland JD, Klaus A, Garratt AN, Birchmeier W: Wnt signaling in stem and cancer stem cells. Curr Opin Cell Biol 2013;25:254-264.

-21 Takebe N, Harris PJ, Warren RQ Ivy SP: Targeting cancer stem cells by inhibiting wnt, notch, and hedgehog pathways. Nat Rev Clin Oncol 2011;8:97-106.

22 Amiel E, Everts B, Freitas TC, King IL, Curtis JD, Pearce EL, Pearce EJ: Inhibition of mechanistic target of rapamycin promotes dendritic cell activation and enhances therapeutic autologous vaccination in mice. J Immunol 2012;189:2151-2158.

23 Sarkar S, Korolchuk VI, Renna M, Imarisio S, Fleming A, Williams A, Garcia-Arencibia M, Rose C, Luo S, Underwood BR, Kroemer G, O'Kane CJ, Rubinsztein DC: Complex inhibitory effects of nitric oxide on autophagy. Mol Cell 2011;43:19-32. 